\title{
Modelling a cage mooring system with pull-back chains for industrial aquaculture
}

\author{
Alexey Trifanov ${ }^{1}$, Evgeny Tikhonov $^{2}$, Valentin Bazykin ${ }^{1} *$ \\ ${ }^{1}$ Federal Scientific Agroengineering Centre VIM, 3, Fil'trovskoye sh., 196625, pos. Tyarlevo, Saint- \\ Petersburg, Russia \\ ${ }^{2}$ Petrozavodsk State University, 33, pr. Lenina, 185910, Petrozavodsk, Russia
}

\begin{abstract}
This paper discusses the technical aspects of modeling the cage retention system for industrial fish farming in a pond using pull chains. The purpose of this work: substantiation of the parameters of the holding ropes, the length of the chains and the required mass of the anchors by calculating the tensile forces in the ropes and the reaction of the anchor supports when solving the dynamic problem in an unsteady setting. Dynamic system was developed that takes into account wind, wave and inertial loads acting on all elements of the system: cage, holding ropes, chains, anchors. The force acting on the surface of the cage from the wind is received. For this, a solid-state cage model was developed with equivalent windage. The tensile forces in the ropes and the reaction of the anchor supports in three planes are obtained. The lengths of the chains are selected, ensuring the absence of tearing forces acting on the anchors. Moreover, the calculations were performed in a non-stationary setting with a gradual increase in load. The simulation duration was $60 \mathrm{~s}$. During this time, one resonance phenomenon was revealed: a significant increase in the breaking strength in the rope at 37 seconds of calculation.
\end{abstract}

\section{Introduction}

Currently, the Republic of Karelia produces more than 27 thousand tons of commercial trout. At the same time, the Republic of Karelia has a huge potential for intensive growth of production volumes. Estimated production volumes can reach 35 thousand tons per year. A number of other regions in the world are also increasing industrial production of commercial trout. At the same time, active work is underway to justify the cultivation of trout in Central Russia, where conditions are not very favorable. In General, industrial fish farming in many countries is a priority for the development of food production. With the growth of fish production, the issues of technological, technical, and environmental issues related to the safe production of industrial fish farming products are becoming more acute.

This paper discusses the technical issues of calculating and designing a cage positioning system for industrial fish farming. A widespread, "Norwegian positioning system" [1], the principle of which is based on the use of round-link chains connecting the holding rope and the anchor and the placement of cages in cells formed by the holding ropes [2]. In this case,

\footnotetext{
*Corresponding author: valentin-bazykin@mail.ru
} 
the forces acting on the cage are transmitted to the holding ropes through intermediate ropes that connect the cage to the holding ropes. At the same time, if the cage module consists of only one cage, such a system turns out to be unreasonably expensive due to the large number of ropes and anchors. In this case, it is more rational to attach the cage directly to the holding ropes connected to the anchors. As an alternative to the classic fishbreeding cages, the development and implementation of underwater-based cages is underway $[3,4]$. Underwater-based technology has a serious problem: refill fish swim bladders necessarity. This challenge also was tried to solve [5]. However, in the Republic of Karelia, this technology requires at least a medium-term perspective for the development of technologies for industrial trout cultivation in open reservoirs. In general, this direction of development is observed all over the world $[6,7]$.

\section{Materials and methods}

The purpose of this work is to justify the parameters of the holding ropes, the length of the chains and the required weight of the anchors by calculating the tensile forces in the ropes and the reactions of the anchors supports.

To achieve this goal, we need to solve the following problem: develop a dynamic system that takes into account wind, wave and inertial loads that affect all elements of the system: the cage, holding ropes, chains, and anchors [8].

To solve this problem, we used numerical modeling methods, specifically the finite element method in a non-stationary formulation. This method is using for such kind of issues [8], in different forms of models $[9,10]$.

The first stage of solving this problem was the development of a positioning system for a $25 \mathrm{~m}$ diameter cage. General layout is shown in figure 1 .

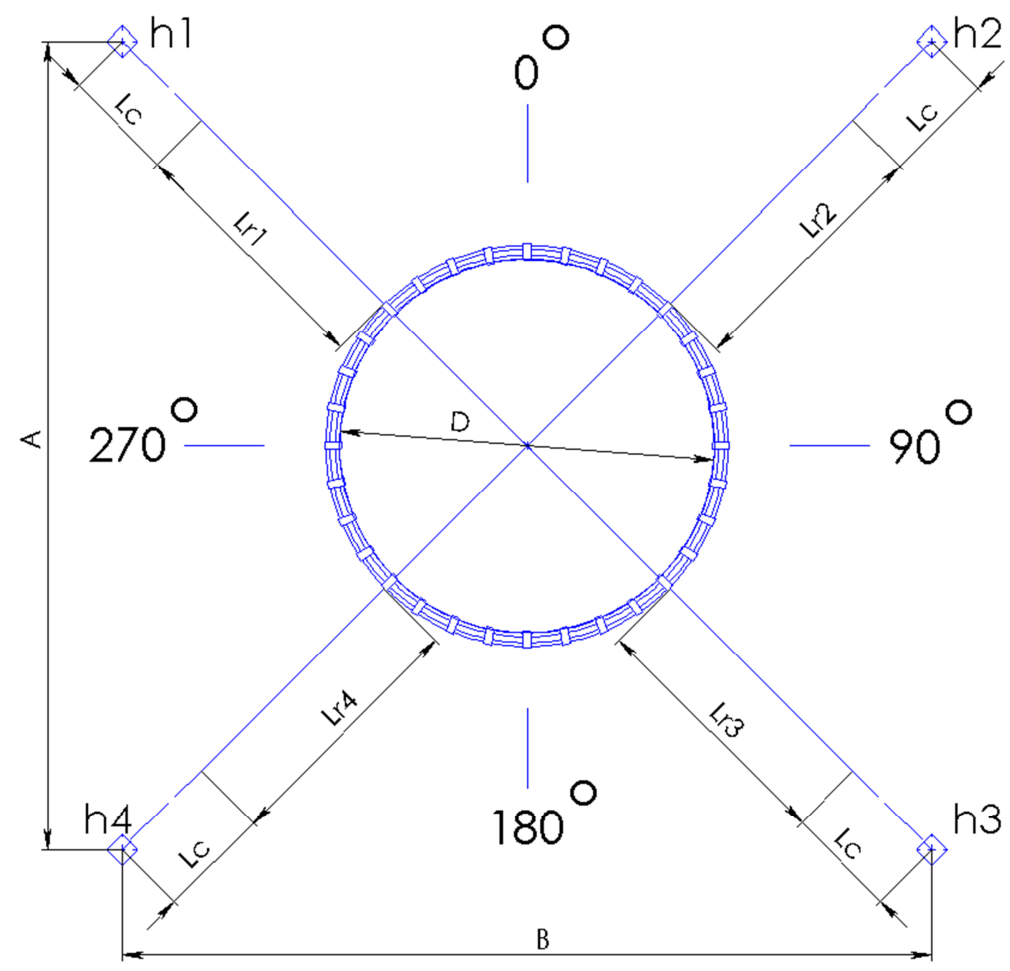

Fig. 1. Cage positioning system. 
The data required to perform the calculation is shown in table 1. This type of data is equal like in other investigations in this topic $[11,12]$.

Table 1. The data required to perform the calculation.

\begin{tabular}{|c|c|c|}
\hline № & Parameter & Value \\
\hline \multicolumn{3}{|c|}{ Outer loads } \\
\hline 1 & Wind velocity, $\mathrm{m} / \mathrm{sec}$ & 25 \\
\hline 3 & Wind direction, deg. (see fig. 1) & 0 \\
\hline 4 & Wave height, $\mathrm{m}$ & 2.4 \\
\hline 5 & Wave speed, $\mathrm{m} / \mathrm{sec}$ & 4.5 \\
\hline 6 & Wave length, $\mathrm{m}$ & 33 \\
\hline 7 & Wave direction, deg. (see fig. 1) & 0 \\
\hline \multicolumn{3}{|c|}{ Cage parameters } \\
\hline 1 & Internal diameter $(\mathrm{S}), \mathrm{m}$ & 25 \\
\hline 2 & Tubes diameter, $\mathrm{mm}$ & 315 \\
\hline 3 & Distance between tubes, $\mathrm{mm}$ & 550 \\
\hline 4 & Rail diameter, $\mathrm{mm}$ & 90 \\
\hline 5 & Rail height, mm & 950 \\
\hline \multicolumn{3}{|c|}{ Positioning system parameters (see fig. 1) } \\
\hline 1 & System width (A), m & 60 \\
\hline 2 & System length (B), m & 60 \\
\hline 3 & Chain length $(\mathrm{Lc}), \mathrm{m}$ & 12.5 \\
\hline 4 & Main rope length $(2 \mathrm{Lr}+\mathrm{D}), \mathrm{m}$ & 175 \\
\hline 5 & Mass of $1 \mathrm{~m}$ chain, $\mathrm{kg}$ & 44 \\
\hline 6 & Anchor material & Granite \\
\hline \multicolumn{3}{|c|}{ Calculation parameters } \\
\hline 1 & Modelling duration, sec & 59 \\
\hline 2 & Safety coefficient for ropes & 1.4 \\
\hline
\end{tabular}

The stages of solving the task are as follows [13]:

1. Determination of the dynamic wind load on the elements of the surface part of the cage;

2. Determination of cage movements from the impact of dynamic wave load;

3 . The calculation of the maximum rupture loads on the containment ropes;

4. Calculation of the required weight of the anchor, taking into account the density of the anchor material.

To perform the above steps, we use FlowSimulation and Simulation software packages. The use of these software packages shows good adequacy of the calculation models and coordination of the calculation results with the results of field studies [14]. In order to reduce the resource intensity and the calculation time, we use an adapted cage model with an equivalent windage (see fig. 2).

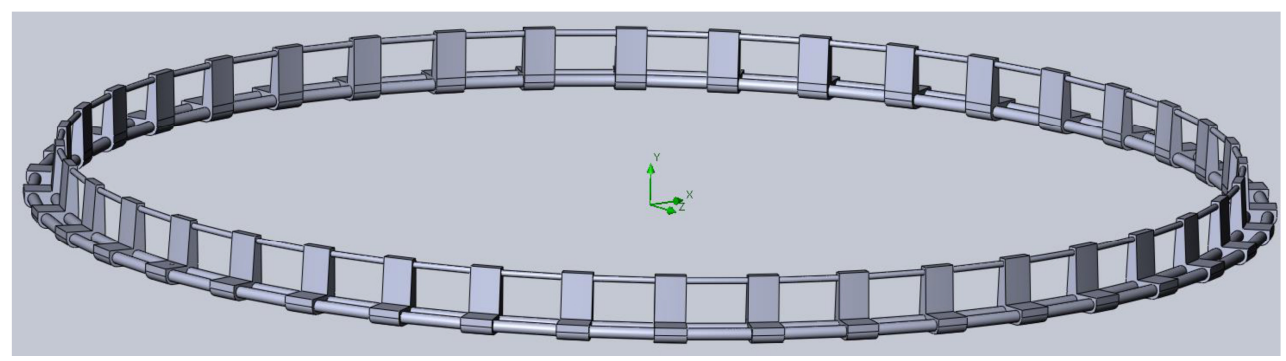

Fig. 2. The modified cage model for wind loads calculation.

The result of the aerodynamic calculation is shown in figure 3 . 


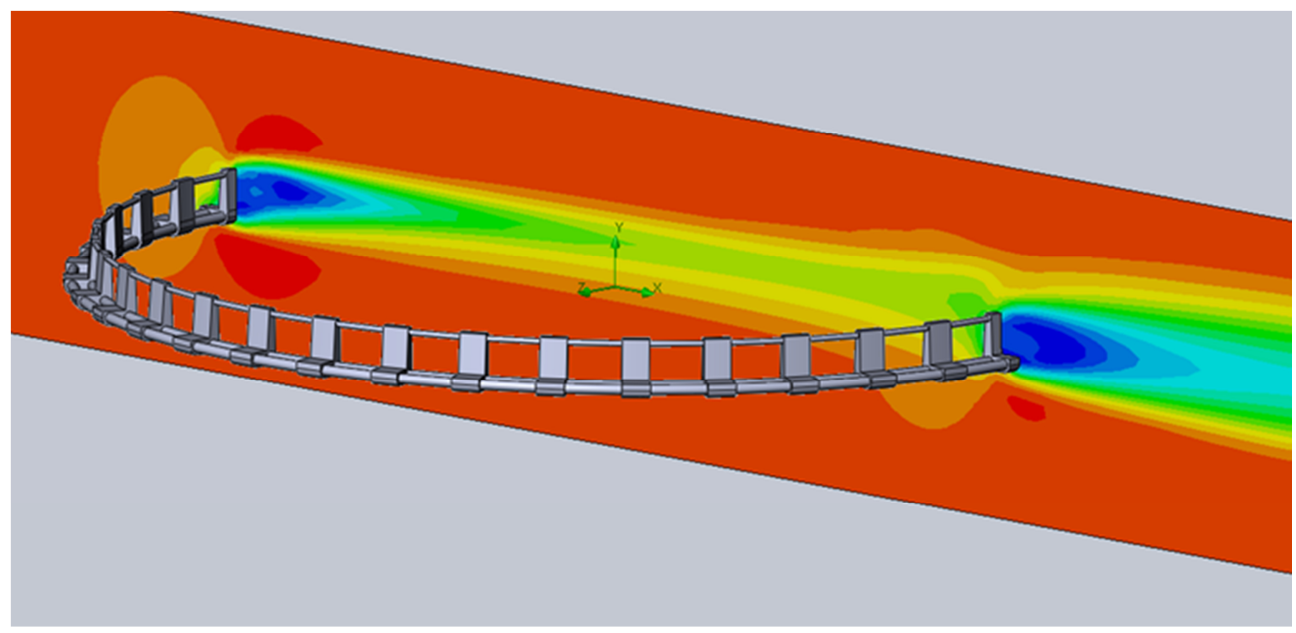

Fig. 3. Plot of air flows velocities acting on the cage.

The impact of air flows leads to elastic deformations of the buoyancy elements of the cage. The cage takes the form of an ellipse. The reaction of the support of the attachment point of the cage will give the total force from the pressure of the air masses on the elements of the cage structure (see fig. 4).

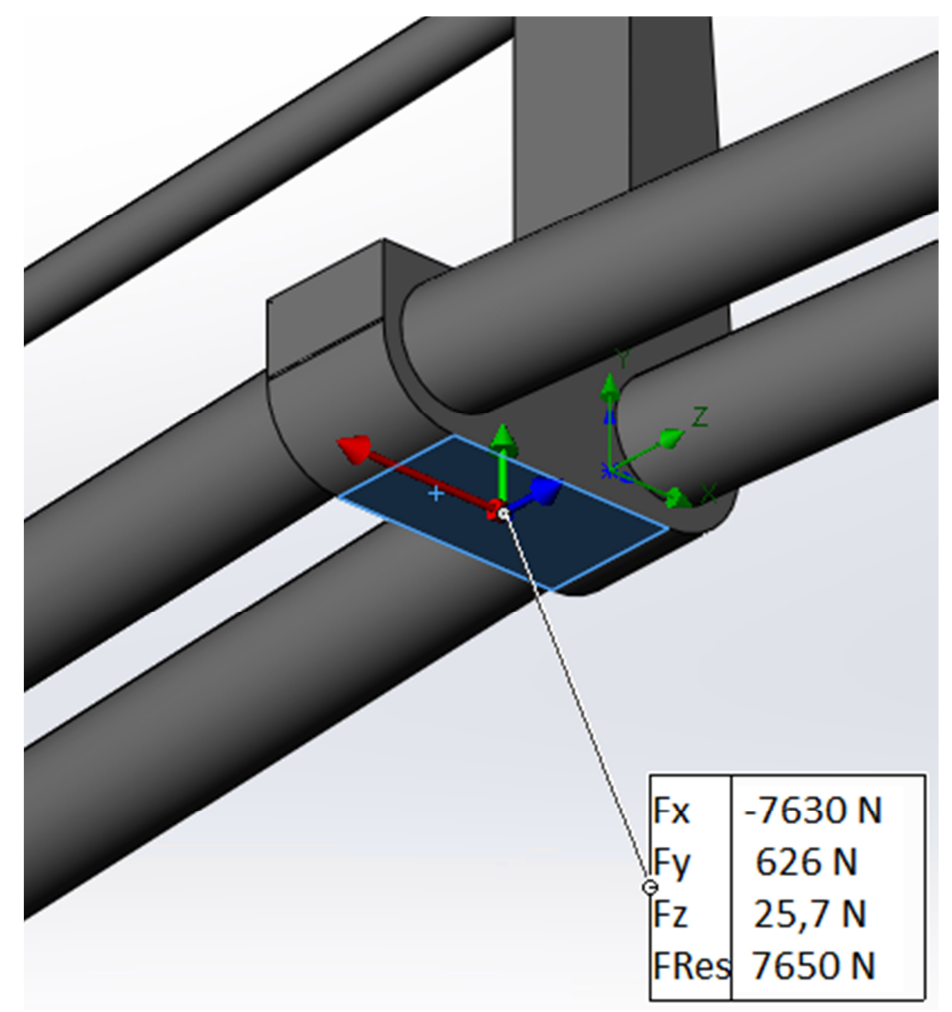

Fig. 4. Air mass pressure force acting on the cage.

For the next stages of the calculation, take the wind load equal to $7650 \mathrm{~N}$.

The next stage of the calculation: determining the movements of the cage from the impact of dynamic wave load, we use the ANSYS software package. To build the 
calculation model, we use the fundamental model of the cage [15]: a square-shaped surface with a diagonal equal to the outer diameter of the cage, with points for attaching ropes (see fig. 5). As a rope models, we use the link180 beam element [16]. The calculation model of the cage positioning system is shown in figure 6.

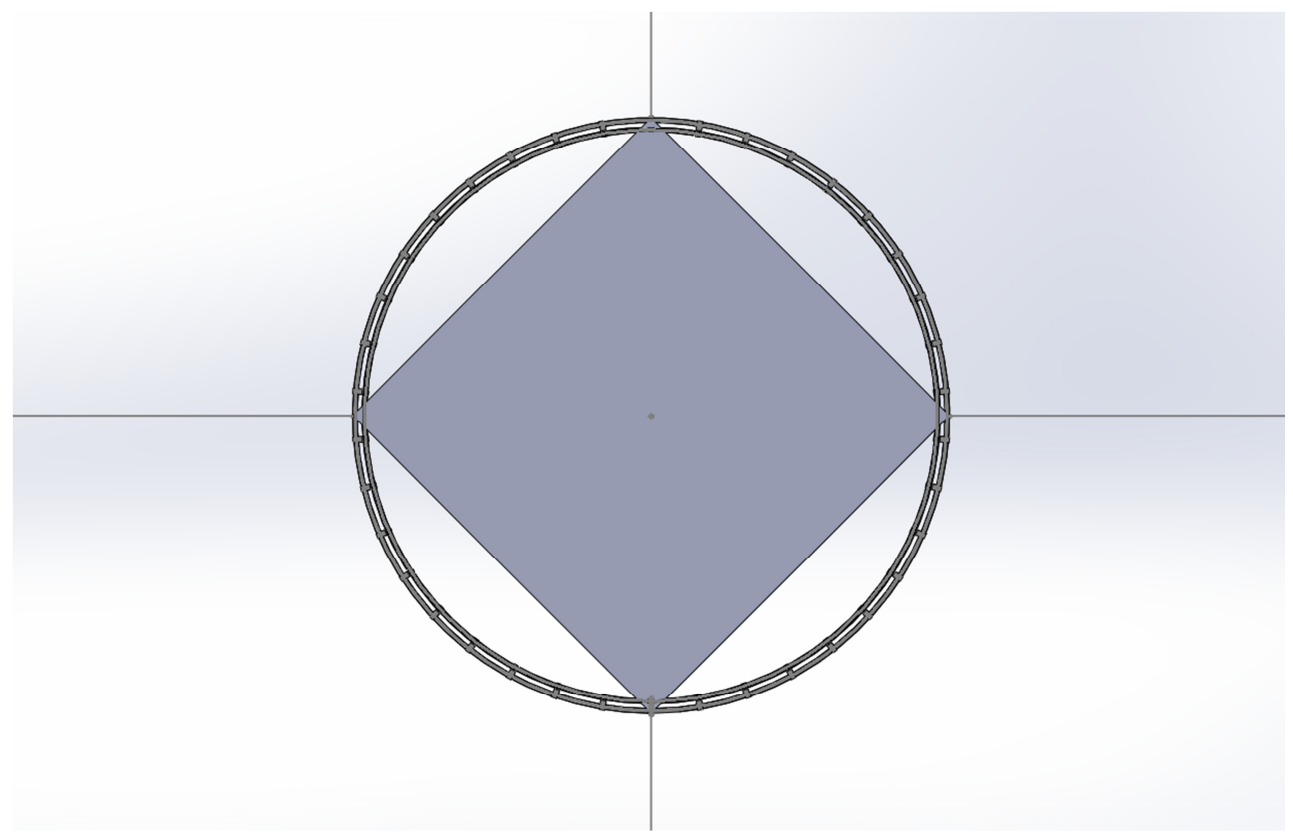

Fig. 5. Fundamental model of the cage.

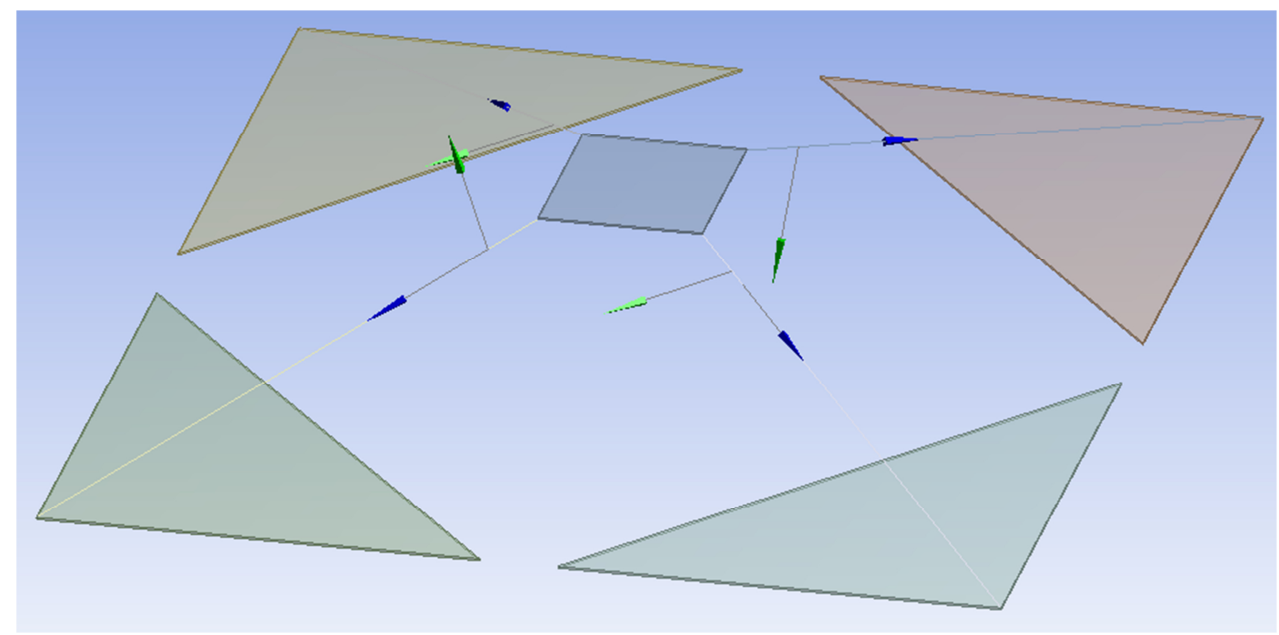

Fig. 6. General view of the calculation model.

In figure 6, triangles form part of the bottom of the reservoir. Chains will be placed on these surfaces when the cage is moved vertically.

The vertical movement of the cage is determined by the shape of the water surface. The shape of the water surface is formed by a wave with the parameters specified in the initial data (table. 1). In the calculation model, the wave is defined by a harmonic function with the corresponding amplitude and frequency [17]. 
In accordance with the cage positioning scheme, the calculation model is limited to 4 anchor points. The wind load is applied to the cage (see fig. 7 and 8).

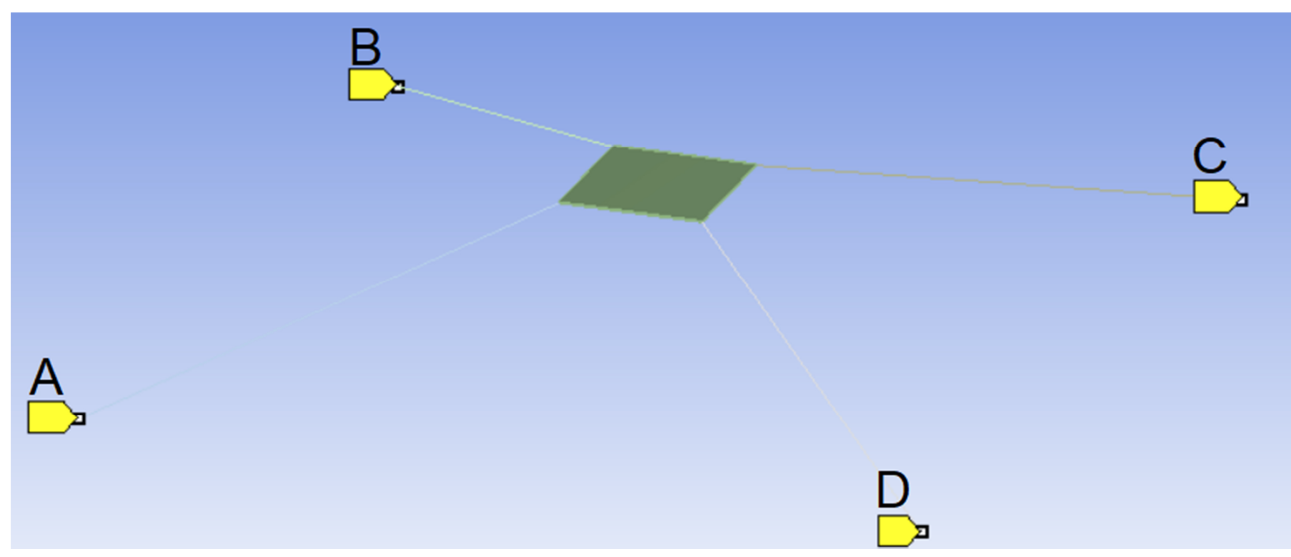

Fig. 7. Anchors (A, B, C, D).

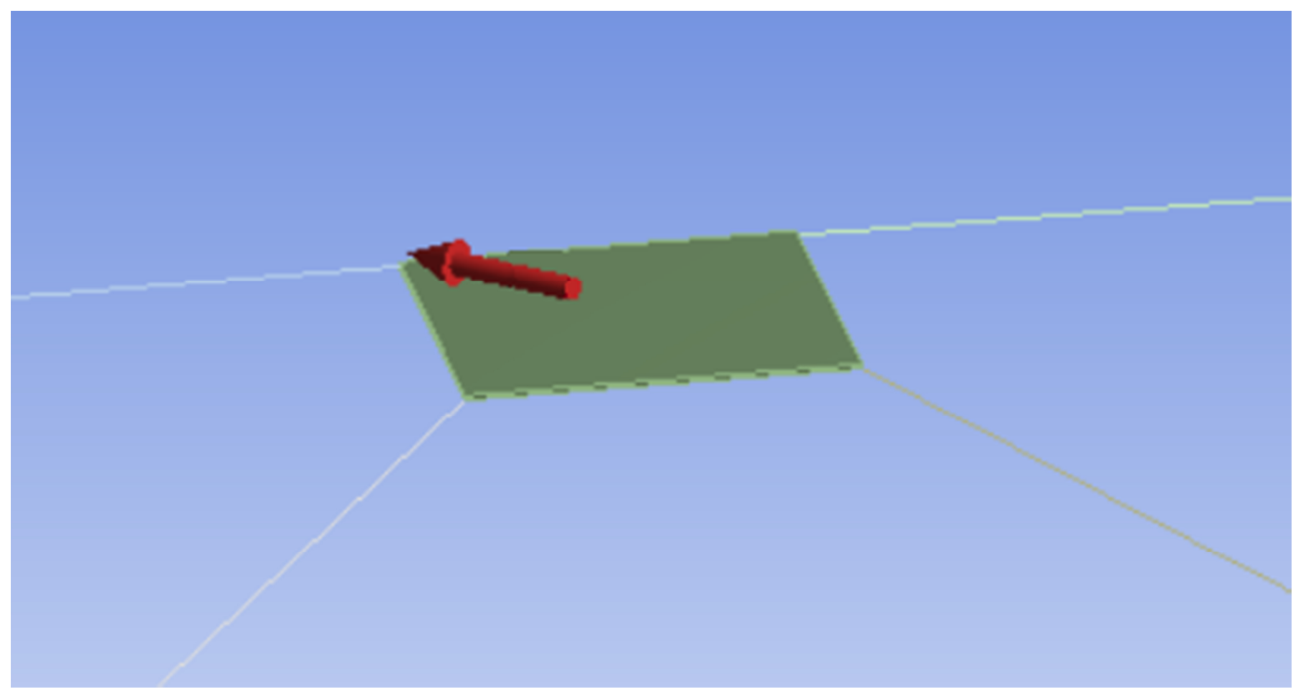

Fig. 8. Wind load vector.

The direction of movement of the waves is collinear with the wind load vector.

The gravity in the calculation model is reflected by an acceleration equal to 9,807 $\mathrm{m} / \mathrm{sec}^{2}$. The estimated system simulation time is 59 seconds, and the minimum step in the calculation time is 0,02 seconds.

\section{Results and discussion}

As the simulation results, graphs of rupture forces of the holding ropes are obtained. The graph of the rupture force of the most loaded rope is shown in figure 9. 


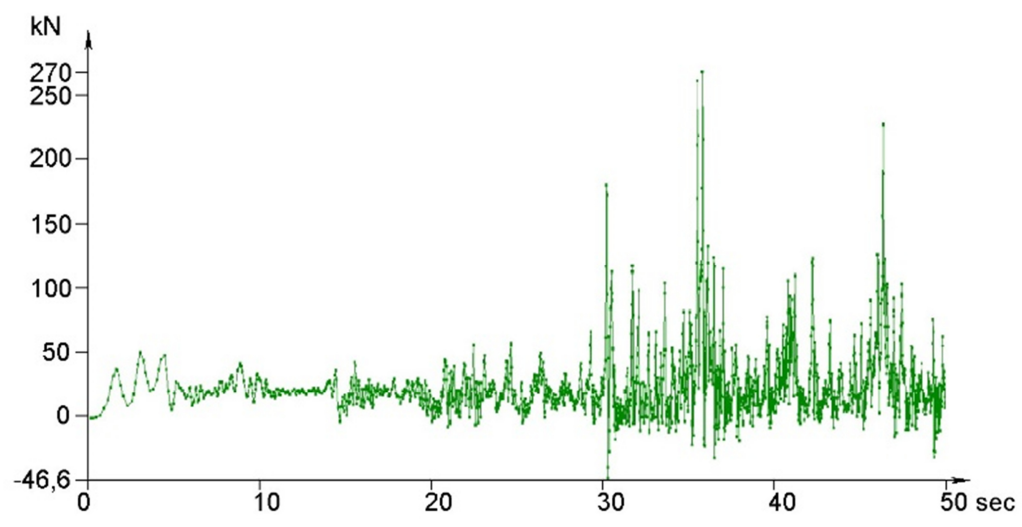

Fig. 9. Graph of the rupture force of the most loaded rope (N).

The greatest rupture force acting on the rope and equal to $270 \cdot 10^{3}$ is fixed at 36 seconds of simulation. At this point, the phenomenon of system resonance occurs, due to the cyclical nature of external and inertial loads of the system.

To calculate the weight of anchor, it is necessary to take into account the ratio of the vertical component of the anchor support reaction to the horizontal components $-\mathrm{Z} / \mathrm{XY}$. This $\mathrm{Z} / \mathrm{XY}$ ratio takes into account the effect of reducing the friction force between anchor and the bottom. Table 2 shows the reaction values of the anchors supports on Cartesian axes.

Table 2. Maximum reactions of supports along the Cartesian axes.

\begin{tabular}{|c|c|c|c|c|}
\hline $\begin{array}{c}\text { № of } \\
\text { anchor }\end{array}$ & $\begin{array}{c}\text { Axis X, } \\
\mathbf{N}\end{array}$ & $\begin{array}{c}\text { Axis Z, } \\
\mathbf{N}\end{array}$ & $\begin{array}{c}\text { Axis Y, } \\
\mathbf{N}\end{array}$ & $\begin{array}{c}\text { Horizontal component of } \\
\text { the reaction, } \overrightarrow{\boldsymbol{X}}+\overrightarrow{\boldsymbol{Y}}, \mathbf{H}\end{array}$ \\
\hline 1 & $2.51 \cdot 10^{5}$ & 68888 & 8422,3 & 261136 \\
\hline 2 & 13605 & 58805 & $1,99 \cdot 10^{5}$ & 199478 \\
\hline 3 & $1.7 \cdot 10^{5}$ & 51628 & 8565,5 & 180204 \\
\hline 4 & 16614 & 51930 & $2.17 \cdot 10^{5}$ & 217643 \\
\hline
\end{tabular}

The next step in the calculation is to determine the gravity of the anchors. The required gravity force of each anchor is performed by calculating the required friction force between the anchor and the bottom. The friction coefficient is taken as for the anchor, without hooks, paws and sandy bottom: $f=1$. The friction force is calculated by the formula:

$$
F_{f r}=f \cdot N
$$

$N$ - support reaction (the force of gravity), $\mathrm{N}$.

Take into account the decrease in the gravity force from the vertical component of the support reaction (see table. 3 ):

$$
F_{f r}=f \cdot\left(N+N_{z}\right)
$$

$N_{Z}$ - support reaction along $Z$ axis, $N$.

Calculate the anchor gravity force:

$$
N=\frac{F_{f r}}{f}+N_{z}
$$

Therefore, the minimum required gravity force of the anchor will be: 


$$
N=\frac{\vec{X}+\vec{Y}}{f}+N_{z}
$$

The calculation results are summarized in table 3 .

Table 3. Minimum required gravity force of anchor in water.

\begin{tabular}{|c|c|}
\hline № of anchor & Gravity force, $\mathbf{k N}$ \\
\hline 1 & 330 \\
\hline 2 & 258.3 \\
\hline 3 & 231.8 \\
\hline 4 & 269.6 \\
\hline
\end{tabular}

The calculated gravity force acts in the water. In order to calculate the required mass of anchor, it is necessary to calculate the Archimedean force acting on anchors in water. To do this, take the density of the anchor material (granite) equal to $2600 \mathrm{~kg} / \mathrm{m}^{3}$. The force of Archimedes is calculated using the formula:

$$
F_{\text {Arch }}=\rho_{\mathrm{H}_{2} \mathrm{O}} \cdot g \cdot V
$$

$\rho_{\mathrm{H} 2 \mathrm{O}}-$ water density, $\mathrm{kg} / \mathrm{m}^{3}$;

$\mathrm{g}-$ acceleration of gravity, $\mathrm{m} / \mathrm{c}^{2}$;

$\mathrm{V}$ - anchor volume, $\mathrm{m}^{3}$.

The required gravity force of the anchor acting on land will be equal to:

$$
F_{\text {land }}=F_{\mathrm{H}_{2} \mathrm{O}}+\rho_{\mathrm{H}_{2} \mathrm{O}} \cdot g \cdot V
$$

The final calculated weight of the anchors is calculated using the formula:

$$
M_{\text {anchor }}=\frac{F_{\text {land }}}{g}
$$

Next, perform the calculation of the masses of granite anchors. The results are shown in table. 4.

Table 4. The estimated masses of the anchors.

\begin{tabular}{|c|c|}
\hline № of anchors & Calculated anchor masses, ton. \\
\hline 1 & 53.7 \\
\hline 2 & 42 \\
\hline 3 & 37.7 \\
\hline 4 & 43.9 \\
\hline
\end{tabular}

\section{Conclusion}

Modeling the positioning system of a single cage according to the scheme proposed above shows significant loads on the holding ropes and large calculated weights of the anchors. This is primarily due to the high wave loads adopted in the model. The results of the calculation show the principal possibility of implementing the positioning systems of the cage modules according to the proposed scheme. The main advantage is to reduce the number of ropes and the required rigging equipment. The main drawback of the calculated system is that it cannot be used in conditions of heavy wave and wind loads, since the required rope diameters and cargo weights are obtained significantly. Not all industrial aquaculture enterprises have the necessary lifting equipment. However, the use of such positioning systems for cage modules will be acceptable under conditions of small and medium wave and wind loads. 
In the future, the main challenges of aquaculture will be in offshore systems, where the wave and wind loads are very serious. This conditions will require new calculation models based on real experiments.

\section{References}

1. Aqualine Midgard System, http://www.aqualine.no/en/products/

2. X. Qu, F. Hu, T. Kumazawa, Y. Takeuchi, T. Tokai, Ocean Engineering 17, 619-624 (2019) https://doi.org/10.1016/j.oceaneng.2018.12.016

3. A. Ton $\mathrm{Nu}$ Hai, S. Speelman, Marine Policy 11, 103799 (2020) https://doi.org/10.1016/j.marpol.2019.103799

4. A. Drach, I. Tsukrov, J. DeCew, M. Robinson Swift, B. Celikkol, C.F. Hurtado, MARINE (2013)

5. A. Glaropoulosab, L.H. Stiena, O. Folkedala, T. Dempsterc, F. Oppedal, Aquaculture 502, 332-337 (2019) https://doi.org/10.1016/j.aquaculture.2018.12.065

6. B.H. Buck, M.F. Troell, G. Krause, D.L. Angel, B. Grote, T. Chopin, Front. Mar. Sci. (2018) https://doi.org/10.3389/fmars.2018.00165

7. Y. Wang, J. Hu, H. Pan, S. Li, P. Failler, Marine Policy 64, 135-147 (2016) https://doi.org/10.1016/j.marpol.2015.11.014

8. P. Klebert, P. Lader, L. Gansel, F. Oppedal, Ocean Engineering 58, 260-274 (2013) https://doi.org/10.1016/j.oceaneng.2012.11.006

9. T.-J. Xu, Y.-P. Zhao, G.-H. Dong, Y.-C. Li, F.-K. Gui, Journal of Fluids and Structures 39, 222-236 (2013) https://doi.org/10.1016/j.jfluidstructs.2013.02.011

10. G.-H. Dong, M.-F. Tang, T.-J. Xu, C.-W. Bi, W.-J. Guo, Applied Ocean Research 87, 233-246 (2019) https://doi.org/10.1016/j.apor.2019.04.005

11. Y.-H. Kim, D.-S. Whang, Fisheries Research 106, 71-75 (2010) https://doi.org/10.1016/j.fishres.2010.07.005

12. M.-F. Tang, G.-H. Dong, T.-J. Xu, Y.-P. Zhao, W.-J. Guo, Applied Ocean Research 79, 253-261 (2018) https://doi.org/10.1016/j.apor.2018.08.009

13. T. Kristiansen, O.M. Faltinsen, Journal of Fluids and Structures 34, 218-235 (2012) https://doi.org/10.1016/j.jfluidstructs.2012.04.001

14. D.W. Fredriksson, J. DeCew, P. Lader, Z. Volent, Ø. Jensen, F.V. Willumsen, Ocean Engineering 83, 99-110 (2014) https://doi.org/10.1016/j.oceaneng.2014.03.005

15. H. Chen, E. Damgaard Christensen, Ocean Engineering 142, 597-615 (2017) https://doi.org/10.1016/j.oceaneng.2017.07.033

16. H. Moe, A. Fredheim, O.S. Hopperstad, Journal of Fluids and Structures 26, 503-516 (2010) https://doi.org/10.1016/j.jfluidstructs.2010.01.007

17. Y. Yao, Y. Chen, H. Zhou, H. Yang, Journal of Fluids and Structures 62, 350-366 (2016) https://doi.org/10.1016/j.jfluidstructs.2016.01.004 